\title{
Early detection of diabetic neuropathy by investigating CNFL and IENFD in thy1-YFP mice
}

\author{
Janine Leckelt', Pedro Guimarães ${ }^{2}$, Annett Kott' ${ }^{1}$, Alfredo Ruggerí2 ${ }^{2}$, Oliver Stachs ${ }^{3}$ \\ and Simone Baltrusch'
}

'Institute of Medical Biochemistry and Molecular Biology, University of Rostock, Rostock, Germany 2Department of Information Engineering, University of Padua, Padua, Italy

${ }^{3}$ Department of Ophthalmology, University of Rostock, Rostock, Germany

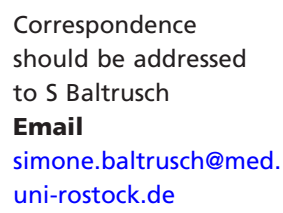

\begin{abstract}
Small fiber neuropathy is one of the most common and painful long-term complications of diabetes mellitus. Examination of the sub-basal corneal nerve plexus is a promising surrogate marker of diabetic neuropathy. To investigate the efficacy, reliability and reproducibility of in vivo corneal confocal microscopy (IVCCM), we used thy1-YFP mice, which express yellow fluorescence protein (YFP) in nerve fibers. 4 weeks after multiple low-dose injections of streptozotocin, thy1-YFP mice showed manifest diabetes. Subsequent application of insulin-releasing pellets for 8 weeks resulted in a significant reduction of blood glucose concentration and $\mathrm{HbA}_{1 c^{\prime}}$ a significant increase in body weight and no further increase in advanced glycation end products (AGEs). IVCCM, carried out regularly over 12 weeks and analyzed both manually and automatically, revealed a significant loss of corneal nerve fiber length (CNFL) during diabetes manifestation and significant recovery after insulin therapy. Ex vivo analyses of CNFL by YFP-based microscopy confirmed the IVCCM results (with high sensitivity between manual and automated approaches) but demonstrated that the changes were restricted to the central cornea. Peripheral areas, not accessible by IVCCM in mice, remained virtually unaffected. Because parallel assessment of intraepidermal nerve fiber density revealed no changes, we conclude that IVCCM robustly captures early signs of diabetic neuropathy.
\end{abstract}

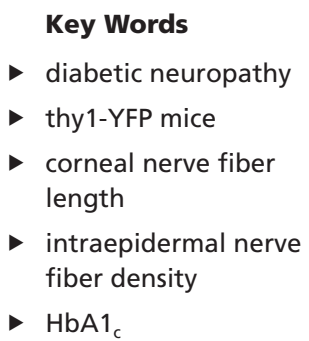

Journal of Endocrinology (2016) 231, 147-157

\section{Introduction}

Diabetic neuropathy, which develops in up to $50 \%$ of patients with type 1 and type 2 diabetes mellitus, represents the third most prevalent neurological disorder and is the primary risk factor for foot ulceration, frequently resulting in amputation. Nerve conduction velocity is nowadays an established clinical routine method to diagnose diabetic neuropathy. However, this technique only recognizes large nerve fiber dysfunction at the end stage of diabetes complications. Although quantitative sensory testing enables to evaluate small nerve fiber function, this non-invasive technique also fails to yield adequate results because of low reproducibility and high subjectivity.

In vivo confocal microscopy, simply based upon reflected light from tissue, permits high-resolution visualization of cellular ultrastructure. There is growing

Published by Bioscientifica Ltd. 
interest in the visualization and investigation of the corneal nerve fibers that form the sub-basal nerve plexus because these nerve fibers comprise the same type of small nerves, thinly myelinated A-delta and non-myelinated C-fibers, as those present in skin. These corneal nerve fibers are regarded as a potential surrogate marker to represent the peripheral nerve status of patients with diabetes mellitus (Guthoff et al. 2009, Ziegler et al. 2014). In contrast to performing skin biopsies, in vivo corneal confocal microscopy (IVCCM) allows monitoring of diabetic neuropathy in a noninvasive and longitudinal manner. As an ophthalmologic modality, IVCCM is currently emerging as a promising additional diagnostic tool in the assessment of diabetic neuropathy (Rosenberg et al. 2000, Tavakoli et al. 2010, Malik 2014).

To date, however, only a few clinical studies have investigated changes in corneal and intraepidermal nerve fiber density (IENFD) in a parallel manner during diabetes progression and therapy. In particular, knowledge is sparse concerning the capacity for and time course of regeneration of impaired small nerve fibers in skin and cornea (Quattrini et al. 2007, Tavakoli et al. 2013, Ziegler et al. 2014, Azmi et al. 2015). Recent reports of the use of IVCCM in animal studies (Leppin et al. 2014, Ehmke et al. 2015, Yorek et al. 2015) for ex vivo analyses not feasible in humans have provided a framework to review the efficacy, reliability and reproducibility of this technique in the detection of diabetic neuropathy (Islam 2013).

Thy1-YFP mice, which express yellow fluorescent protein (YFP) in nerve fibers, are a suitable animal model in this context. The presence of this marker in thy1-YFP mice permits nerve fiber quantification in both in vivo and ex vivo studies without the need for staining procedures (Chen et al. 2005, Ehmke et al. 2015).

The aim of this study is therefore to investigate the changes in corneal and intraepidermal nerve fiber together with metabolic changes in thy1-YFP mice suffering from streptozotocin (STZ)-induced diabetes and receiving insulin treatment.

\section{Materials and methods}

\section{Animals}

Male homozygote B6.Cg-Tg(Thy1-YFP)16Jrs/J mice were purchased from Jackson Laboratories, bred and housed at the central animal care facility of the Medical Faculty, University of Rostock, and received conventional rodent chow (SSNIFF, Soest, Germany) and water ad libitum. This study was conducted in accordance with the German Animal Welfare Act 2006 (last amended 2014) and was approved by the State Department of Agriculture, Food Safety and Fisheries, Mecklenburg-Vorpommern (LALLF $\mathrm{M}-\mathrm{V})$. Mice aged 10-12 weeks were treated with multiple low-dose intraperitoneal injections of streptozotocin (STZ) $(65 \mathrm{mg} / \mathrm{kg}$; St Louis, MO, USA) $(n=15)$ or an equal volume of vehicle solution $(50 \mathrm{mmol} / \mathrm{L}$ sodium citrate buffer, $\mathrm{pH}$ 4.5) $(n=10)$. Mice with blood glucose levels of $15 \mathrm{mmol} / \mathrm{L}$ or higher were considered diabetic. After 4 weeks of diabetes, insulin-releasing pellets $(0.1 \mathrm{U} / 24 \mathrm{~h}$; LinBit, Scarborough, Canada) were implanted subcutaneously under short-inhalation anesthesia with diethyl ether (Deventer, Netherlands).
A

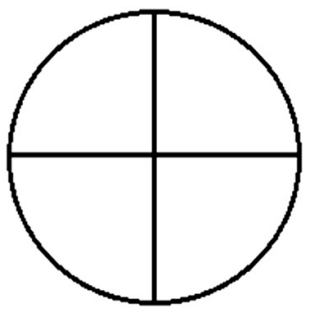

B

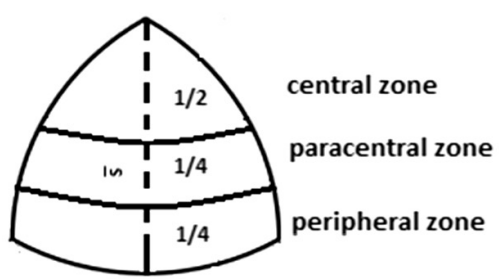

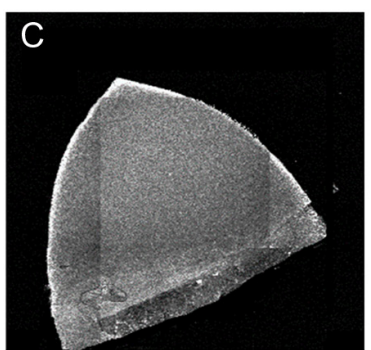

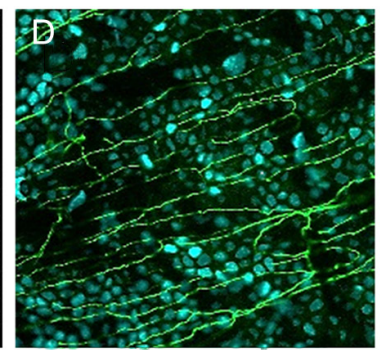

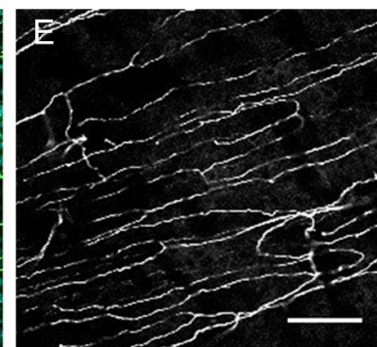

Figure 1

Ex vivo confocal imaging of the thy1-YFP mouse cornea. (A) Cornea divided into superonasal, superotemporal, inferonasal and inferotemporal quadrants. (B) A quadrant separated into peripheral, paracentral and central zones. (C) Overview image of a quadrant. (D) Nerve fibers (green) and epithelial cell nuclei (blue). (E) Sub-basal nerve fibers. Scale bar: $50 \mu \mathrm{m}$. http://joe.endocrinology-journals.org DOI: 10.1530/JOE-16-0284
๑) 2016 Society for Endocrinology Printed in Great Britain
Published by Bioscientifica Ltd. 


\section{Metabolic measurements}

Mice underwent daily blood glucose (Freestyle Mini, Abbott, Germany) and body weight measurements, and $\mathrm{HbA}_{1 \mathrm{c}}$ levels were determined using the $\mathrm{HbA}_{1 \mathrm{c}}$ reagent kit (Tarrytown, NY, USA). Serum samples were assayed to quantify C-peptide values and advanced glycation end product (AGE) levels (mouse C-peptide and mouse AGE ELISA; ALPCO Diagnostics, Salem, NH, USA).

\section{In vivo corneal confocal microscopy}

Initially and then at 2, 4, 6 and 12 weeks after STZinduced diabetes and insulin treatment, IVCCM was performed using the Heidelberg Retina Tomograph II equipped with a Rostock Cornea Module (HRTII/RCM), as described in detail elsewhere (Reichard et al. 2010, 2014). Mice were anesthetized with a subcutaneous injection of $0.5 \mathrm{mg} / \mathrm{kg}$ medetomidine (Dorbene; Léon, Spain) and $5 \mathrm{mg} / \mathrm{kg}$ ketamine (Ketamin Inresa; Neuss, Germany) and were placed in a MouseFix animal holder (Steven GmbH, Ochtrup, Germany) during the examination (15-20 min for both eyes). Using the sequence mode with a frame rate of four frames per second, the central parts of the corneal subepithelial nerve plexus were scanned over an area of approximately $900 \times 900 \mu \mathrm{m}$. Images acquired have a field of view of $300 \times 300 \mu \mathrm{m}$ with a resolution of $384 \times 384$ pixels. From each eye meeting specific criteria, five images (nonoverlapping areas, absence of pressure-induced artifacts, section with the greatest number of visible nerve fibers and nerve fibers with clear structure) were chosen for further quantification. In addition, images were selected in which the same or at least similar regions of nerve fibers were represented in the same animal. Finally, the corneal nerve fiber length (CNFL) was calculated in $\mathrm{mm} / \mathrm{mm}^{2}$ as the total length of all sub-basal nerve fibers and branches. Quantification was performed first using NeuronJ, a semiautomated nerve analysis plug-in program of ImageJ (NIH) (Meijering et al. 2004), and secondly using a fully automated approach developed in the MATLAB computer language (The MathWorks, Natick, MA, USA) (Reichard et al. 2016) for comparison with the manually analyzed images at the main time points (at baseline and after 4 and 12 weeks).

\section{Ex vivo fluorescence microscopy of the cornea}

Mice were killed either 4 weeks after diabetes onset $(n=7)$ or 8 weeks after insulin treatment $(n=6)$. Control mice $(n=5$, each) were prepared at the same time points. Before the eye was enucleated, the upper position of the cornea was marked by a stitch at the corneoscleral limbus. The globes were removed and fixed for $30 \mathrm{~min}$ in $4 \%$ PFA at room temperature. The corneas were extracted, returned to fixative solution for a further $30 \mathrm{~min}$ and finally stored in 30\% sucrose (SigmaAldrich Chemie $\mathrm{GmbH}$ ) with $0.1 \mathrm{M}$ PBS at $4^{\circ} \mathrm{C}$ for no longer than 2 days. The cornea was cut into four equal quadrants, superonasal, superotemporal, inferonasal and inferotemporal, with spring-loaded micro-scissors under a light microscope (Carl Zeiss AG, Jena, Germany) (Fig. 1A) (Dvorscak \& Marfurt 2008). All samples were counterstained with DAPI, mounted with Vecta Shield medium (Vector Laboratories, Peterborough, UK) and analyzed using a Fluoview FV10i confocal laser scanning microscope (Olympus). An automatically generated overview image enabled subdivision of each quadrant
A

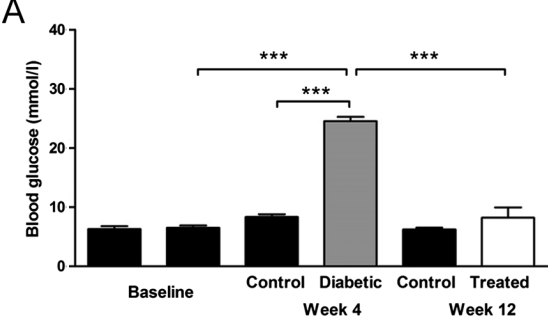

C

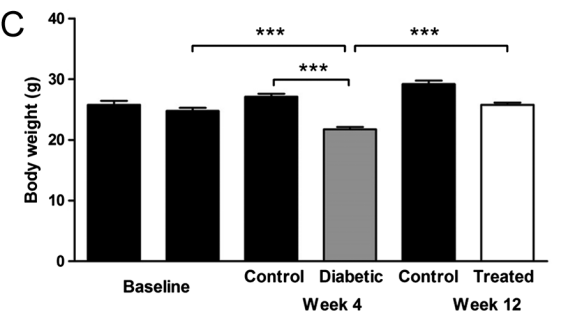

B
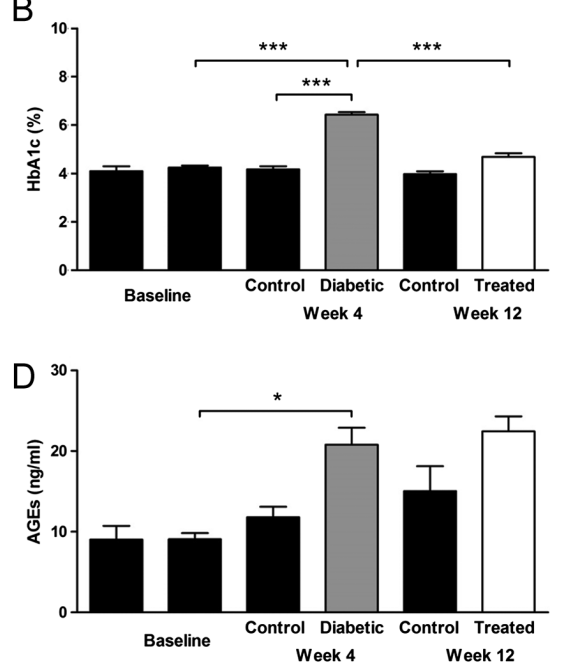

Figure 2

Insulin treatment results in metabolic normalization of STZ-diabetic thy1-YFP mice. Blood glucose (A), $\mathrm{HbA}_{1 \mathrm{c}}(\mathrm{B})$, body weight (C) and AGEs (D) in control (black bars), diabetic (gray bars) and treated (white bars) mice at baseline and 4 and 12 weeks of the experiment. Data are expressed as mean \pm S.E.M. from 5 to 15 mice per group. ${ }^{*} P<0.05, * * * P<0.001$ (ANOVA/ Bonferroni correction). http://joe.endocrinology-journals.org DOI: $10.1530 / J O E-16-0284$
(C) 2016 Society for Endocrinology Printed in Great Britain 

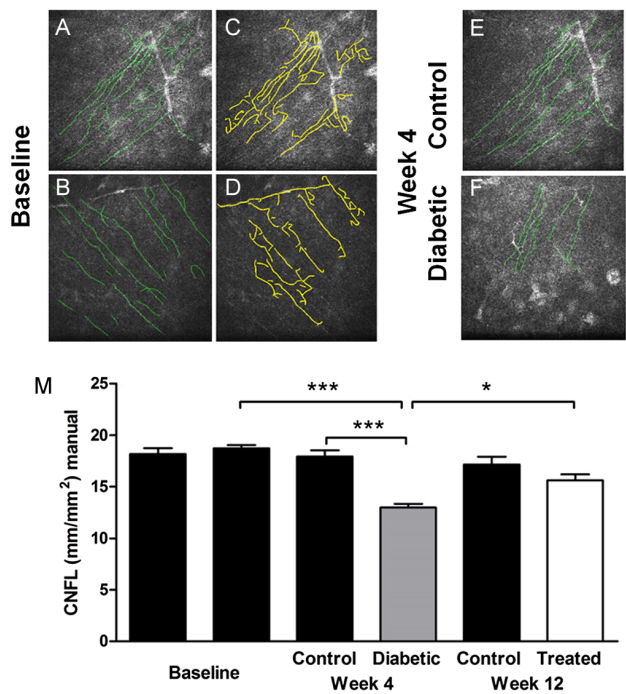
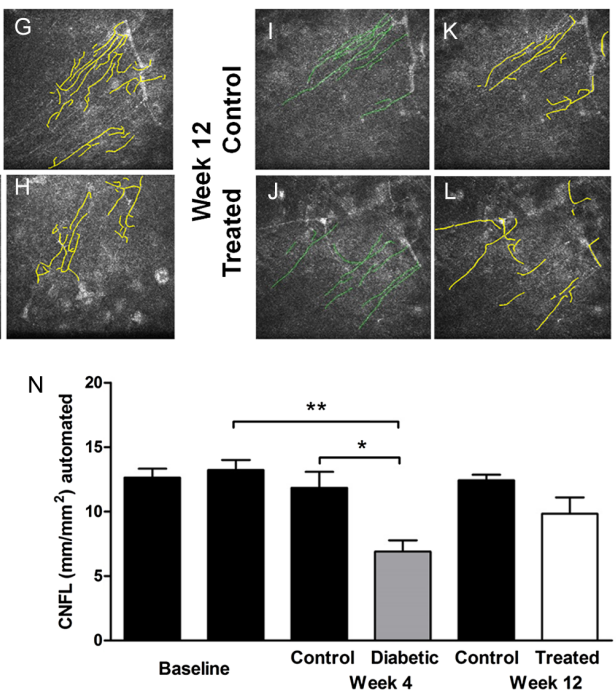
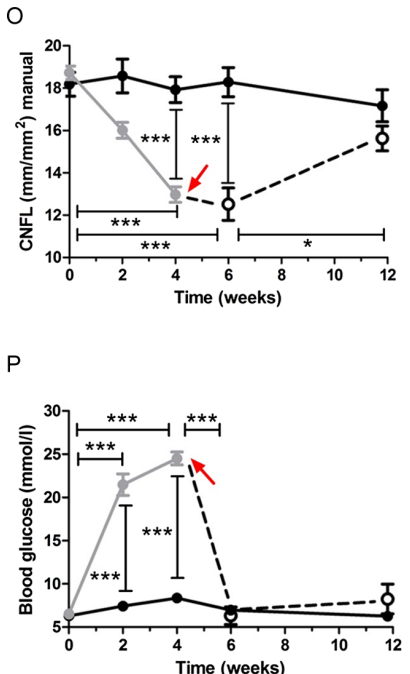

Figure 3

Insulin treatment results in recovery of CNFL in the cornea of STZ-diabetic thy1-YFP mice. CNFL was calculated from IVCCM images, as illustrated, for example, at baseline (A-D) and $4(E-H)$ and $12(I-L)$ weeks of the experiment. CNFL was quantified manually by Image $(A, B, E, F, I, J$, green and $M, O)$ and automatically (C, D, G, H, K, L, yellow and N) in control (black bars and circles), diabetic (gray bars and circles) and treated (white bars and open circles) mice. Blood glucose values are shown for all time points of manually quantified CNFL (P). A red arrow marks the start of insulin treatment. Data are expressed as mean \pm S.E.M. from 5 to 15 mice per group. ${ }^{*} P<0.05,{ }^{*} P<0.01,{ }^{*} * P<0.001$ (ANOVA/Bonferroni correction).

into three different zones: peripheral, paracentral and central (Fig. 1B). Finally, six to ten images each were acquired centrally and peripherally (Fig. 1C and D). Also, five non-overlapping images of YFP fluorescence
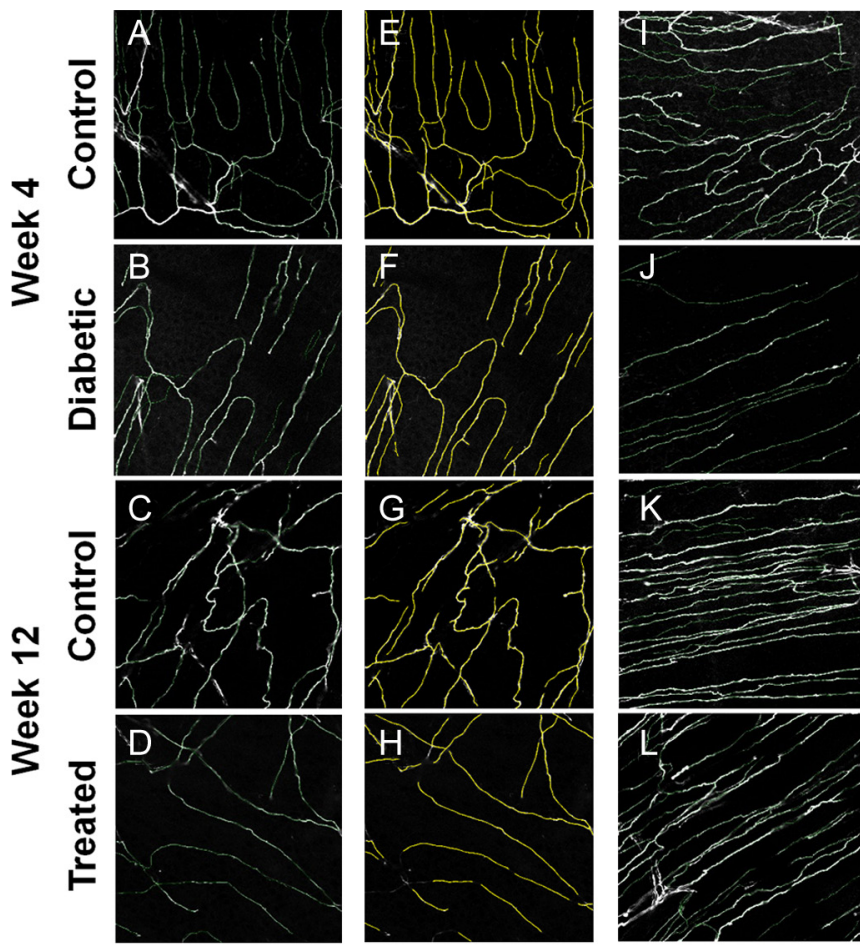

Peripheral zone
$(212 \times 212 \mu \mathrm{M} ; 1024 \times 1024$ pixels $)$ with the greatest number of visible nerve fibers from each zone and quadrant were quantified (Fig. 1E) in the same procedure as described for IVCCM.

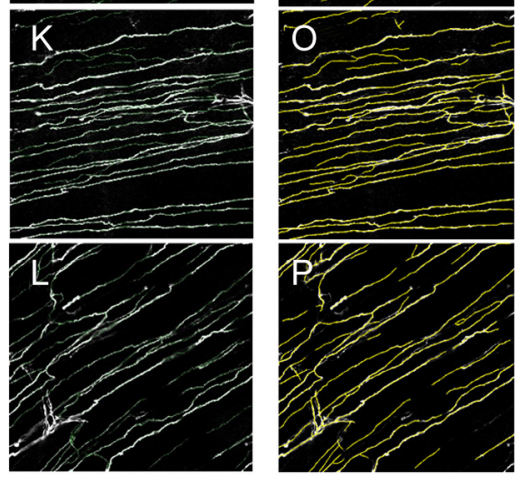

Central zone

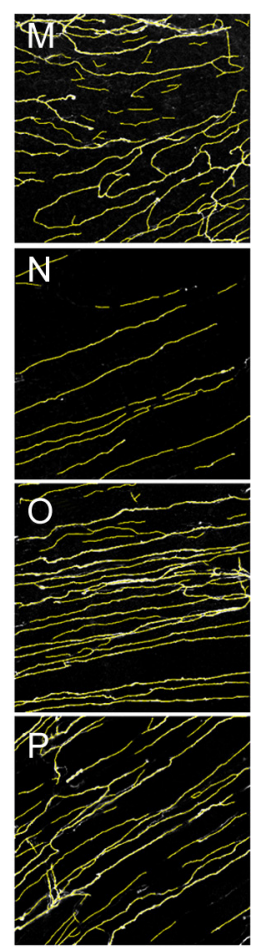

Figure 4

Comparison between manual and automated tracing of nerve fibers in fluorescence images. $E x$ vivo fluorescence images of control $(A, E, I, M, C$, $G, K, O)$, diabetic $(B, F, J, N)$ and treated $(D, H, L$, P) thy1-YFP mice at week $4(A, B, E, F, I, J, M, N)$ and week $12(C, D, G, H, K, L, O, P)$ were analyzed by Image and a manual approach $(A-D, I-L$, green) and by automated tracing of nerve fibers $(\mathrm{E}-\mathrm{H}, \mathrm{M}-\mathrm{P}$, yellow) in the peripheral $(\mathrm{A}-\mathrm{H})$ and central (I-P) zones. http://joe.endocrinology-journals.org
DOI: $10.1530 / J O E-16-0284$
๑๐ 2016 Society for Endocrinology Printed in Great Britain 


\section{Intraepidermal nerve fiber density}

Samples $(4 \times 1 \mathrm{~mm})$ were harvested from the plantar surface of the right hind paw and fixed in 4\% PFA for $4 \mathrm{~h}$, followed by cryoprotection in 20\% sucrose with $0.1 \mathrm{M}$ PBS for $24 \mathrm{~h}$. After embedding with the longitudinal lateral side of the right hind paw in optimal cutting temperature compound (Deventer, Netherlands), tissues were stored at $-80^{\circ} \mathrm{C}$. Ten serial $10 \mu \mathrm{M}$ thick slices per animal were cut perpendicular to the skin surface. Images were counterstained overnight at $4^{\circ} \mathrm{C}$ with rabbit polyclonal antibody against PGP9.5 (1:500, ab10404; Abcam, Hamburg, Germany) (Beiswenger et al. 2008, Ehmke et al. 2015, Reichard et al. 2016) followed by secondary antibody goat antirabbit-TRITC (1:500, ab50598; Abcam) for $1 \mathrm{~h}$ at room temperature. All samples were counterstained with DAPI and mounted with Vecta Shield medium. Three sections from each animal were randomly analyzed using the FV10i (Olympus). An overview of the epidermis was reconstructed by acquisition of z-stacks with a total depth of $0.5 \mu \mathrm{M}$ in $15-20$ overlapping images. In line with guidelines published by the European Federation of Neurological Societies (Lauria et al. 2010), intraepidermal nerve fibers crossing the
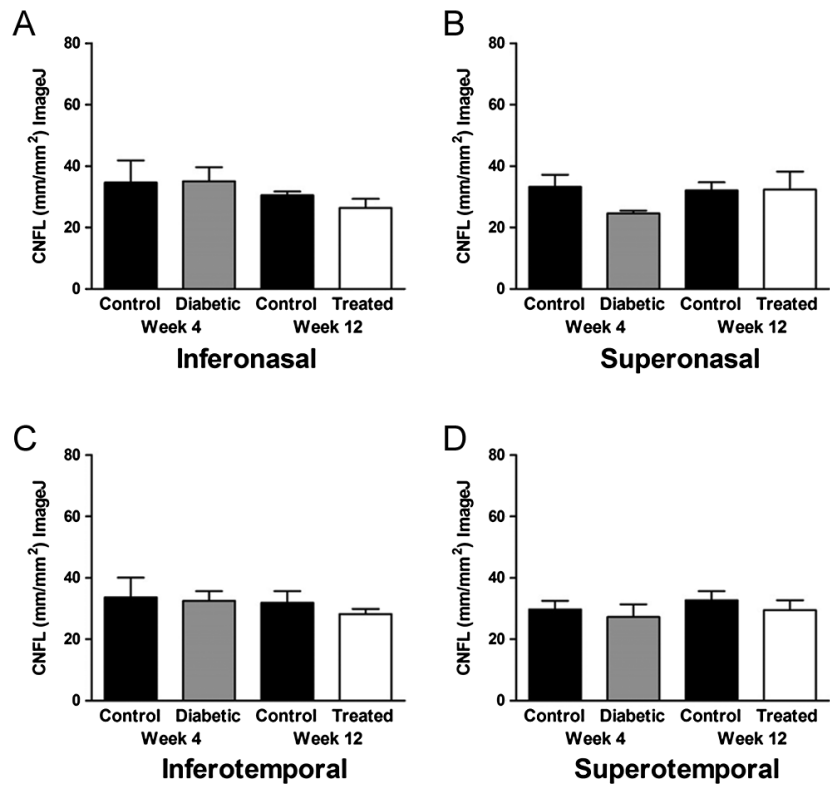

Peripheral zone dermal-epidermal border were counted along the length of the epidermis using ImageJ. IENFD was expressed as the number of nerve fibers per millimeter epidermis.

\section{Multiphoton microscopy of the cornea}

Mice were killed and positioned on a heating plate to maintain body temperature of $37^{\circ} \mathrm{C}$. The eye was analyzed using an upright FVMPE-RS multiphoton microscope (Olympus) equipped with a femtosecond laser system (InSight DS Dual-OL; Spectra-Physics, Santa Clara, CA, USA) and an XLPLN25xWMP (NA 1.05) objective (Olympus). Vidisic eye gel (Bausch \& Lomb GmbH, Berlin, Germany) was used as an immersion medium to avoid desiccation of the eye. YFP was excited at a wavelength of $1040 \mathrm{~nm}$ and, because of their strong auto-fluorescence, the corneal epithelium and endothelium were excited at $740 \mathrm{~nm}$. Images were analyzed with FV30S (Olympus) and Imaris (Bitplane, Zurich, Switzerland).

\section{Statistical analysis}

Statistical analyses were performed using the Prism analysis program (GraphPad, San Diego, CA, USA) and
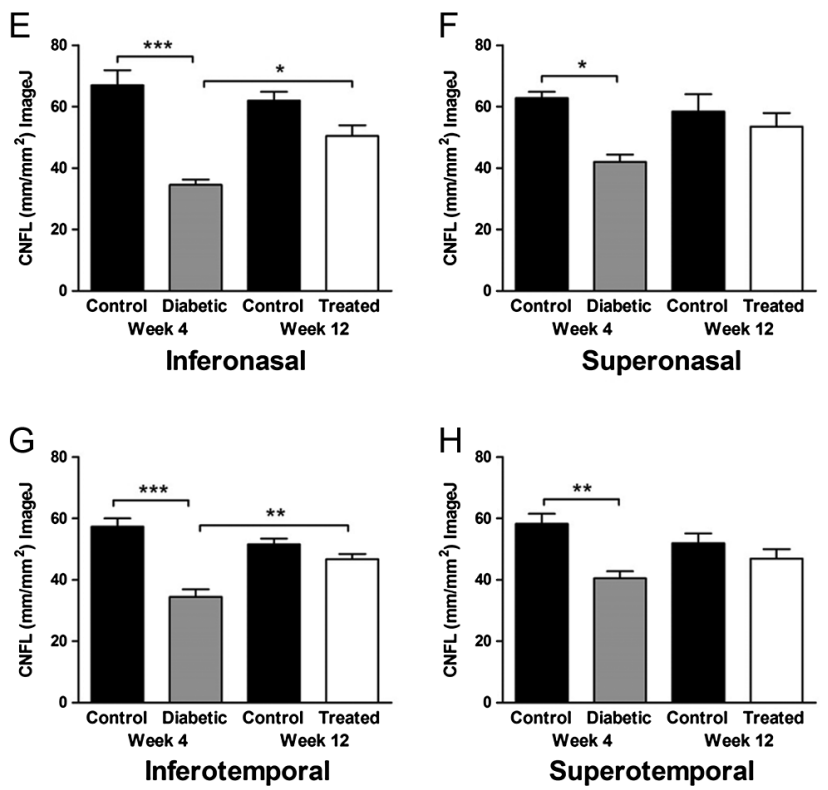

Central zone

\section{Figure 5}

Small fiber nerve damage due to diabetes and recovery on insulin therapy occurred exclusively in the central and not in peripheral corneal zone. CNFL in control (black bars), diabetic (gray bars) and treated (white bars) mice at 4 and 12 weeks of the experiment in inferonasal ( $A$, E), superonasal (B, F), inferotemporal $(C, G)$ and superotemporal $(D, H)$ quadrants in the peripheral $(A-D)$ and central $(E-H)$ zones. Data are expressed as mean \pm S.E.M. from four to six mice per group. ${ }^{*} P<0.05, * * P<0.01, * * * P<0.001$ (ANOVA/Bonferroni correction).

http://joe.endocrinology-journals.org DOI: 10.1530/JOE-16-0284
๑) 2016 Society for Endocrinology Printed in Great Britain
Published by Bioscientifica Ltd. 
the results are presented as mean \pm S.E.M. Differences between diabetic and control mice were examined using Student's $t$-test, and between control, diabetic and insulin-treated mice were examined using ANOVA/ Bonferroni correction. $P$ values $<0.05$ were considered to be statistically significant.

\section{Results \\ Effects of diabetes and subsequent insulin treatment on metabolic parameters in thy1-YFP mice}

Thy1-YFP mice showed comparable baseline values for blood glucose, $\mathrm{HbA}_{1 \mathrm{c}}$, body weight and AGEs (Fig. 2). After 4 weeks of diabetes, STZ-treated thy1-YFP mice showed a significant increase in blood glucose (Fig. 2A), $\mathrm{HbA}_{1 \mathrm{c}}$ (Fig. 2B) and AGEs (Fig. 2D) and a decrease in body weight (Fig. 2C). Compared with control mice at the same time point, significant differences were observed for blood glucose, $\mathrm{HbA}_{1 \mathrm{c}}$ and body weight (Fig. 2). Reduced C-peptide values $(0.81 \pm 0.22$ vs $1.83 \pm 0.25 \mathrm{ng} / \mathrm{mL}, P<0.02$, data not shown) confirmed STZ-induced insulin deficiency in diabetic mice. Following 4 weeks of STZ diabetes, thy1-YFP mice were treated with five insulin-releasing pellets for 8 weeks. This therapy resulted in significant normalization of blood glucose (Fig. 2A) and $\mathrm{HbA}_{1 \mathrm{c}}$ (Fig. 2B) without any significant differences from control mice at the same time point. In addition, treated mice showed significant body weight gain (Fig. 2C), but not fully up to the level of control mice, which showed an age-dependent increase in body weight. AGEs of thy1-YFP mice also displayed an age-dependent increase (Fig. 2). The observed absence of a further increase in AGEs following insulin application to STZ-diabetic mice here can therefore be considered as a positive outcome of treatment.

\section{IVCCM images of effects of diabetes and subsequent insulin treatment on corneal nerves in thy1-YFP mice}

STZ-diabetic mice showed a significant decrease in CNFL (Fig. 3M) after 4 weeks (Fig. 3F) compared with both baseline (Fig. 3B) and time-matched controls (Fig. 3E). Subsequent insulin treatment led to a significant increase (Fig. 3M) in CNFL (Fig. 3J). At week 12, CNFL was lower in insulin-treated mice compared with controls (Fig. 3I), but not significantly (Fig. 3M). The increase in CNFL after 8 weeks of insulin treatment (Fig. 3O) followed the normalization of blood glucose after 2 weeks (Fig. 3P).
For CNFL, the effects of time, treatment and interaction were significant (two-way ANOVA, $P<0.001$, data not shown). There was a moderate negative correlation between $\mathrm{CNFL}$ and $\mathrm{HbA}_{1 \mathrm{c}}$ (linear regression analysis, $R^{2}=0.66, P<0.001$, data not shown) and a weak negative correlation between CNFL and AGE levels (linear regression analysis, $R^{2}=0.33, P<0.01$, data not shown).
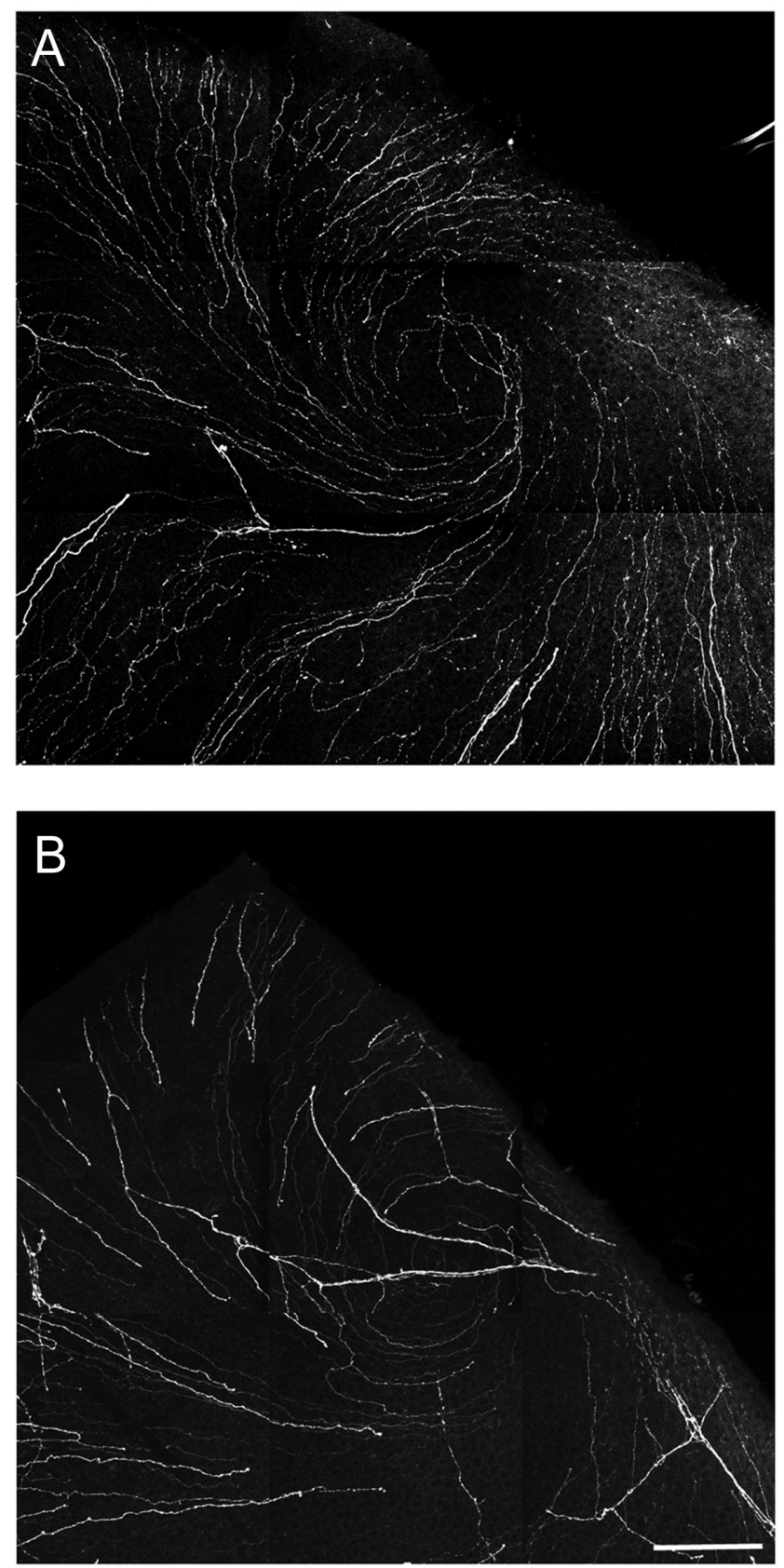

Figure 6

Mapped images of the inferior whorl of corneal small nerve fibers using ex vivo YFP fluorescence in a control (A) and diabetic (B) thy1-YFP mouse. Scale bar: $100 \mu \mathrm{m}$.

Published by Bioscientifica Ltd. 

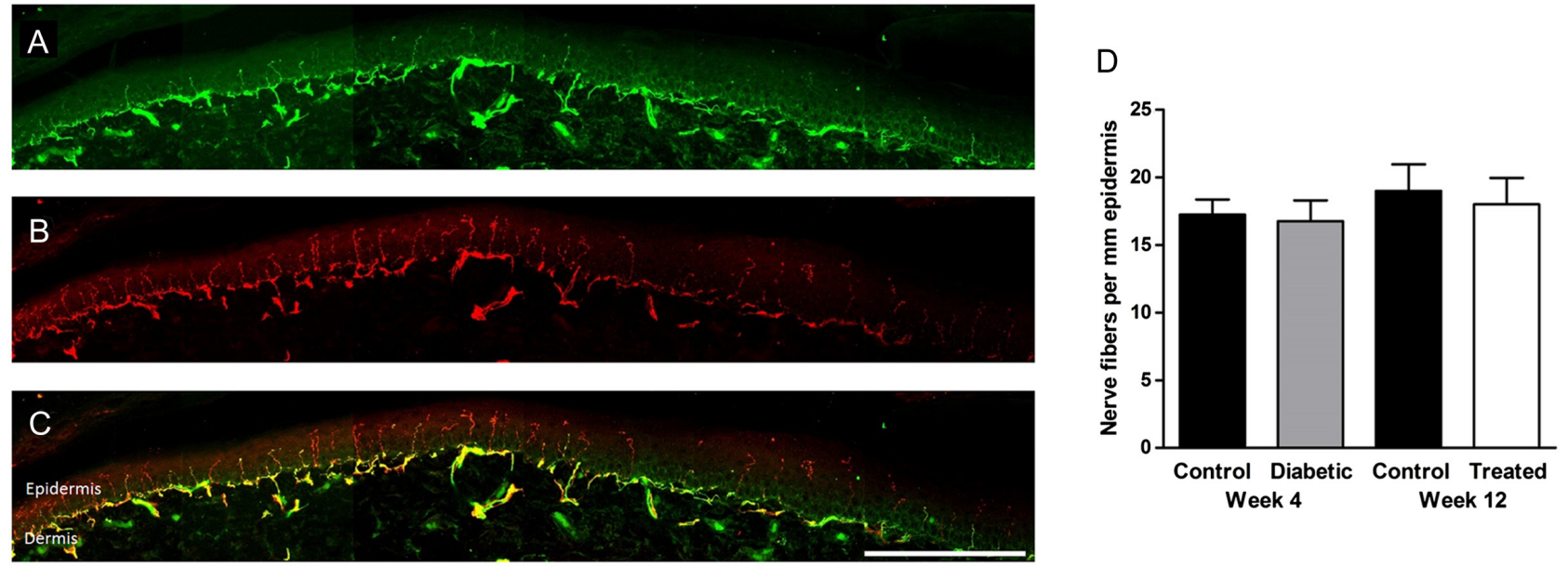

Figure 7

(A) Intraepidermal nerve fiber density did not change after 4 weeks of diabetes in thy1-YFP mice. An illustrative example of intraepidermal nerves is shown in a control mouse. The merged image (C) demonstrates the good correlation between YFP fluorescence (A) and PGP9.5 staining (B). Scale bar: $200 \mu \mathrm{m}$. $D$ : Data are expressed as mean \pm S.E.M. from four mice per group.

\section{Comparison of manual and automated analysis of IVCCM images}

Baseline CNFL in the sub-basal nerve plexus determined by the manual computer-aided method was approximately $18.19 \mathrm{~mm} / \mathrm{mm}^{2}$ for the control group and $18.73 \mathrm{~mm} / \mathrm{mm}^{2}$ for the group later given STZ (Fig. 3A, B and M). With the fully automated method, baseline CNFL was also comparable (12.63 vs $13.10 \mathrm{~mm} / \mathrm{mm}^{2}$ ), but lower (Fig. 3C, $\mathrm{D}$ and $\mathrm{N})$. Diabetes induction reduced CNFL significantly by $27 \%$ ( 17.93 vs $13.18 \mathrm{~mm} / \mathrm{mm}^{2}$, Fig. 3B, F and M) with the manual method and by $36 \%$ (11.83 vs $7.56 \mathrm{~mm} / \mathrm{mm}^{2}$, Fig. 3D, $\mathrm{H}$ and $\mathrm{N}$ ) with the automated method. After insulin therapy, CNFL was lower, but not significantly, in the treated group compared with controls, both with the manual (17.17 vs $15.30 \mathrm{~mm} / \mathrm{mm}^{2}$, Fig. 3I, J and M) and the fully automated methods $\left(12.42 \mathrm{vs} 9.83 \mathrm{~mm} / \mathrm{mm}^{2}\right.$, Fig. 3K, L and N).

\section{Ex vivo microscopy of the corneal sub-basal nerve plexus using YFP}

CNFL was determined in thy1-YFP mice in different areas of the cornea (Figs 4 and 5). In the central zone, CNFL was approximately twice than that in the peripheral zone (Figs 4 and 5). Manually quantified images (Fig. 4A-D, I-L) were also analyzed with the automated software (Fig. 4E-H, M-P). Comparison of the two methods revealed a mean sensitivity of $88 \pm 3 \%$ and a mean false discovery rate of $13 \pm 2 \%$. The four corneal quadrants in control mice did not differ in CNFL, either peripherally (inferonasal: $34.64 \mathrm{~mm} / \mathrm{mm}^{2}$; inferotemporal: $33.68 \mathrm{~mm} / \mathrm{mm}^{2}$; superonasal: $33.21 \mathrm{~mm} / \mathrm{mm}^{2}$; superotemporal: $29.70 \mathrm{~mm} / \mathrm{mm}^{2}$; Fig. $5 \mathrm{~A}-\mathrm{D}$ ) or centrally (inferonasal: $66.94 \mathrm{~mm} / \mathrm{mm}^{2}$; inferotemporal: $57.31 \mathrm{~mm} / \mathrm{mm}^{2}$; superonasal: $62.83 \mathrm{~mm} / \mathrm{mm}^{2}$; superotemporal: $58.23 \mathrm{~mm} / \mathrm{mm}^{2}$; Fig. $\left.5 \mathrm{E}-\mathrm{H}\right)$. In the peripheral zone, CNFL was comparable between diabetic and control thy1-YFP mice (Fig. 5A-D). Centrally, however, a significant decrease in CNFL was observed for all quadrants (inferonasal: $34.52 \mathrm{~mm} / \mathrm{mm}^{2}$; inferotemporal: $34.48 \mathrm{~mm} / \mathrm{mm}^{2}$; superonasal: $41.97 \mathrm{~mm} / \mathrm{mm}^{2}$; superotemporal: $40.49 \mathrm{~mm} / \mathrm{mm}^{2}$; Fig. $5 \mathrm{E}-\mathrm{H}$ ), with the most marked reduction occurring in the inferior quadrants (Fig. 5E and G), and thus affecting the inferior whorl (Fig. 6). After insulin treatment, CNFL remained unaffected in the peripheral zone (Fig. 5A-D), but increased significantly in the central zone (inferonasal: $48.71 \mathrm{~mm} / \mathrm{mm}^{2}$; inferotemporal: $46.75 \mathrm{~mm} / \mathrm{mm}^{2}$; superonasal: $53.54 \mathrm{~mm} / \mathrm{mm}^{2}$; superotemporal: $46.94 \mathrm{~mm} / \mathrm{mm}^{2}$; Fig. 5E-H).

\section{IENFD in thy1-YFP mice}

IENFD did not change in our experiment (Fig. 7). Notably, all nerve fibers expressing YFP (Fig. 7A and C) were positive for PGP9.5 (Fig. 7B and C). No significant changes in IENFD were detected between control, diabetic and treated mice (Fig. 7D).
๑) 2016 Society for Endocrinology Printed in Great Britain 

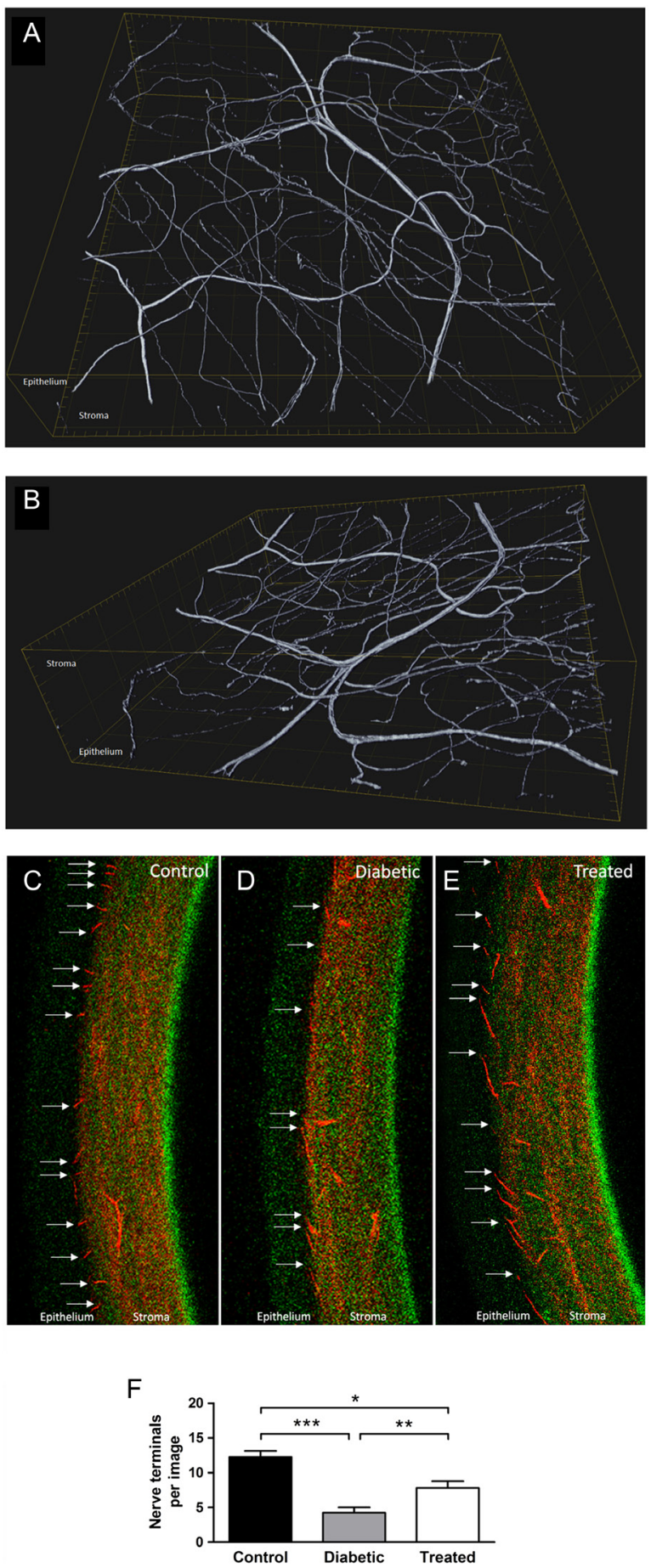

Figure 8

Visualization of beaded and terminal corneal nerve fibers by multiphoton microscopy. Three-dimensional front (A) and side (B) view of the cornea of a control mouse shows that small nerve fibers with beaded structure run parallel above and, in isolated cases, beneath stromal bundled nerve fibers in the central cornea. Cross-sectional images of the cornea (C, D, E) allowed quantification of terminal nerve fibers (red, indicated by white arrows) in control (C, black bar $F$ ), diabetic ( $D$, gray bar $F$ ) and treated mice (E, black bar F). (F) Data are expressed as mean \pm s.E.M. from two mice with 16-18 images in total per group. ${ }^{*} P<0.05$, $* * P<0.01$, $* * * P<0.001$ (ANOVA/Bonferroni correction).

\section{Visualization of corneal nerves by two-photon microscopy}

A three-dimensional map of the cornea illustrated the mesh of small nerve fibers with beaded structure in the cornea (Fig. 8A and B). Cross-sectional images permitted clear differentiation between corneal stroma and epithelium and hence visualization of nerve terminals (Fig. 8C-E). Fine terminal nerve fibers penetrating the epithelium (quantified as number per image) were significantly fewer in diabetic compared with control mice, but increased again after insulin treatment (Fig. 8F).

\section{Discussion}

The widely reported development of IVCCM has had a major impact on the diagnosis and monitoring of diabetic neuropathy, a major complication of diabetes mellitus (Cruzat et al. 2010). However, how corneal nerve fibers are able to reflect damage and regeneration of small nerve fibers in other tissues remains an important, unanswered question. Our combination of longitudinal metabolic and IVCCM analyses with ex vivo investigations of small nerve fibers using YFP fluorescence and the pan-neuronal marker PGP9.5 in a mouse model provides answers that are difficult to obtain in human studies.

We demonstrated that 4 weeks of diabetes evoked a significant decrease in CNFL in thy1-YFP mice, whereas intraepidermal nerve fibers showed no signs of damage. STZ-induced diabetes in a 10- to 12-week-old mouse over 4 weeks corresponds to 5 years of poorly managed diabetes in a 20-year-old human. At that age, regenerative capacity is high and manifestation of major complications of diabetes mellitus is rare. However, the decrease in CNFL sounds a warning that high blood glucose is already sufficient to trigger pathways that cause nerve damage. Subsequent insulin treatment resulting in blood glucose normalization led to a significant increase in CNFL, and this observation supports the hypothesis of a direct correlation between CNFL and glycemic control. A neurotropic effect of insulin should be of secondary importance here because administration was systemic and not topical, as reported elsewhere (Chen et al. 2013). In addition, levels of markers of glycation correlated inversely with changes in CNFL. After 8 weeks of insulin treatment, $\mathrm{HbA}_{1 \mathrm{c}}$, which has natural clearance potential, recovered to control values and AGEs did not increase further. The higher AGE value and the non-total recovery of CNFL in insulin-treated diabetic mice compared with controls may indicate some irreversible damage

Published by Bioscientifica Ltd 
that - even with optimal disease management - might trigger complications at a later stage. Thus, IVCCM evidently has the potential to deliver important disease information very early and in a non-invasive manner.

A comparable recovery effect on corneal nerve fibers after systemic insulin treatment has been reported in diabetic patients, but without improvement in intraepidermal nerve fibers (Tavakoli et al. 2013, Azmi et al. 2015). In recently diagnosed type 2 diabetic patients, the correlation between the two parameters was also weak (Ziegler et al. 2014). To date, the reasons why nerve fiber loss in humans appears earlier in the cornea than in the lower limbs (and sometimes vice versa) are unknown; however, this is most likely to be the result of different pathologies of diabetic neuropathy in the two tissues (Ziegler et al. 2014). In our study, no correlation was found between corneal and intraepidermal nerve fiber changes after 4 weeks of STZ-induced diabetes. Our hypothesis that corneal and intraepidermal nerve fiber changes correlate later on - at an advanced stage of diabetes - is supported by investigations in a small cohort of $\mathrm{C} 57 \mathrm{Bl} / 6 \mathrm{~J}$ mice (Yorek et al. 2014, 2015). In that background strain of thy1-YFP mice, the authors demonstrated both corneal and intraepidermal nerve fiber loss after 20 weeks of STZinduced diabetes. In addition, an older study using the more conventional fluorescence approach has revealed diabetes-induced cutaneous nerve fiber loss in thy1-YFP mice (Chen et al. 2005). Formulation of generally valid conclusions is rendered more challenging by the use of different image acquisition and nerve quantification techniques. This is a topic that needs to be specifically addressed in future research.

Although other studies on mice have analyzed one single captured z-stack (Chen et al. 2013) or two highquality selected images (Yorek et al. 2014, 2015), we recorded five IVCCM images in the center of each eye, based on selection criteria from human studies (Winter et al. 2016). In addition, during the experiment, we sought to rediscover identical or at least similar regions of the murine sub-basal nerve plexus. Because of the small planar surface of the murine cornea (approximately $1 \mathrm{~mm}^{2}$ ), slight variations are technically unavoidable (due to differences in microscope objective orientation or in mouse positioning in the animal holder).

CNFL values vary widely in both mice and humans, even though data are age-matched. Manual computeraided quantification seems to hamper comparability between studies. In a recent investigation focusing on changes in CNFL during aging, we demonstrated that a fully automated algorithm can be used to quantify mouse
IVCCM images (Reichard et al. 2016). Simply because of the corneal size, mouse IVCCM images have naturally lower quality compared with human IVCCM images (Reichard et al. 2016), and this complicates quantification. By applying a further improved automated approach in this study, we confirmed all manually detected significant differences with a respectable sensitivity and false discovery rate. Thus, this automated approach will reduce personal bias and save time, ultimately resulting in improved trial outcomes.

Our aim is to expand the view from the center, made possible by IVCCM, to the whole cornea. Thus, we systematically quantified CNFL in the peripheral and central zone of four separate quadrants using YFP microscopy. Approximately 20 or more different transgenic mouse strains express a fluorescent protein under control of the thy 1 promoter gene and differ in the amount of labeled neuron axons (Porrero et al. 2010). However, within a strain, less variation has been reported (Feng et al. 2000). Like other groups, we observed a low level of variance in corneal neuronal fluorescent YFP fibers (Yu \& Rosenblatt 2007, Namavari et al. 2011). To avoid quenching of YFP by fixation (Ehmke et al. 2015), we used a time-shifted approach and investigated all samples immediately after embedding.

Corneal nerve fibers originate from an ophthalmic division of the trigeminal nerve and penetrate the cornea around the limbus in the deep and mid-stroma (Al-Aqaba et al. 2010). Stromal nerve fibers are visible as bundles and run vertically to the epithelium. Single nerve fibers arising from stromal nerve fibers are orientated parallel to the basal epithelium cells, forming a plexus (Muller et al. 1996). In the periphery, single small nerve fibers are irregular in shape and quantitatively fewer than in the center where nerves show a linear and regular structure. In the inferonasal quadrant, subepithelial nerve fibers appear to terminate in a clockwise pattern to form the inferior whorl. This structure described in humans and mice (Ivanusic et al. 2013) was also observed in this study. Animals with irregular nerve structures, namely without an inferior whorl, were excluded from the study to enhance reliability.

We infer that diabetes-induced nerve fiber loss varies significantly between different regions of the cornea. Reduction in CNFL was significant in all central areas of the cornea, being most pronounced in the inferior quadrants where the nerve fibers of the inferior whorl terminate. Previous studies of diabetic neuropathy in humans and rats have confirmed that nerve fiber loss appears earliest in the region of the inferior whorl

Published by Bioscientifica Ltd. 
(Davidson et al. 2014, Pritchard et al. 2015). However, peripheral areas remained virtually unaffected. Both our IVCCM and YFP-based determinations disclosed a small reduction in CNFL in the center of the cornea in the control group within 8 weeks, confirming some age-dependent nerve fiber loss (Reichard et al. 2016). This finding might support the theory of axon loss in the center (where nerve fibers start to ramify) but not in the periphery of the cornea (Christianson et al. 2003). Insulin treatment produced an increase in central CNFL in STZ-diabetic thy1-YFP mice. Confirming our IVCCM results, recovery of nerve fibers up to baseline levels was incomplete. However, it remains to be established whether corneal nerve fibers regenerate by increasing single axon length or by spreading branches, and whether and to what extent this process is dynamic.

Investigations in STZ-diabetic thy1-YFP mice using two-photon microscopy offer a considerable scope for broadening our understanding of corneal nerve morphology as a surrogate marker for diabetic neuropathy. We have demonstrated previously the high signal-to-noise ratio of this technique in determining changes in CNFL (Ehmke et al. 2015). In this study, the beaded structure of the corneal sub-basal nerve fibers was illustrated by a three-dimensional map of the central cornea. Beaded nerve fibers branch and form nerve terminals in the epithelium, where they fulfill important sensory functions. We have also shown that nerve terminals are highly susceptible to hyperglycemia but are able to recover after insulin treatment. The significant reduction in nerve terminals in STZ-diabetic thy1-YFP mice directly supports the quantification of corneal nerve branches by IVCCM in humans.

In conclusion, we have demonstrated in thy1-YFP mice that diabetes affects corneal nerve fibers exclusively centrally but not peripherally. IVCCM is therefore reliable to detect early signs of nerve damage even though intraepidermal nerve fibers in skin remain unaffected. IVCCM, fully automated quantification of CNFL and two-photon microscopy of STZ-diabetic thy1-YFP mice together provide a promising model to evaluate new compounds for the treatment of diabetic neuropathy.

\section{Declaration of interest}

The authors declare that there is no conflict of interest that could be perceived as prejudicing the research reported.

\section{Funding}

This study was funded by the DDG.

\section{Acknowledgements}

The authors are indebted to Mr David Beattie (freelance medical writer/ UK) for editorial assistance in preparing the manuscript for publication. $\mathrm{J} \mathrm{L}$ is grateful to Mrs Jekatherina Asmus for critical discussion during manuscript drafting.

\section{References}

Al-Aqaba MA, Fares U, Suleman H, Lowe J \& Dua HS 2010 Architecture and distribution of human corneal nerves. British Journal of Ophthalmology 94 784-789. (doi:10.1136/bjo.2009.173799)

Azmi S, Ferdousi M, Petropoulos IN, Ponirakis G, Fadavi H, Tavakoli M, Alam U, Jones W, Marshall A, Jeziorska M, et al. 2015 Corneal confocal microscopy shows an improvement in small-fiber neuropathy in subjects with type 1 diabetes on continuous subcutaneous insulin infusion compared with multiple daily injection. Diabetes Care 38 e3-e4. (doi:10.2337/ dc14-1698)

Beiswenger KK, Calcutt NA \& Mizisin AP 2008 Epidermal nerve fiber quantification in the assessment of diabetic neuropathy. Acta Histochemica 110 351-362. (doi:10.1016/j.acthis.2007.12.004)

Chen YS, Chung SS \& Chung SK 2005 Noninvasive monitoring of diabetes-induced cutaneous nerve fiber loss and hypoalgesia in thy1-YFP transgenic mice. Diabetes 54 3112-3118. (doi:10.2337/ diabetes.54.11.3112)

Chen DK, Frizzi KE, Guernsey LS, Ladt K, Mizisin AP \& Calcutt NA 2013 Repeated monitoring of corneal nerves by confocal microscopy as an index of peripheral neuropathy in type- 1 diabetic rodents and the effects of topical insulin. Journal of the Peripheral Nervous System 18 306-315. (doi:10.1111/jns5.12044)

Christianson JA, Riekhof JT \& Wright DE 2003 Restorative effects of neurotrophin treatment on diabetes-induced cutaneous axon loss in mice. Experimental Neurology 179 188-199. (doi:10.1016/S00144886(02)00017-1)

Cruzat A, Pavan-Langston D \& Hamrah P 2010 In vivo confocal microscopy of corneal nerves: analysis and clinical correlation. Seminars in Ophthalmology 25 171-177. (doi:10.3109/08820538.2010. 518133)

Davidson EP, Coppey LJ, Kardon RH \& Yorek MA 2014 Differences and similarities in development of corneal nerve damage and peripheral neuropathy and in diet-induced obesity and type 2 diabetic rats. Investigative Ophthalmology \& Visual Science 55 1222-1230. (doi:10.1167/iovs.13-13794)

Dvorscak L \& Marfurt CF 2008 Age-related changes in rat corneal epithelial nerve density. Investigative Ophthalmology \& Visual Science 49 910-916. (doi:10.1167/iovs.07-1324)

Ehmke T, Leckelt J, Reichard M, Weiss H, Hovakimyan M, Heisterkamp A, Stachs O \& Baltrusch S 2015 In vivo nonlinear imaging of corneal structures with special focus on BALB/c and streptozotocindiabetic Thy1-YFP mice. Experimental Eye Research 146 137-144. (doi:10.1016/j.exer.2015.11.024)

Feng G, Mellor RH, Bernstein M, Keller-Peck C, Nguyen QT, Wallace M, Nerbonne JM, Lichtman JW \& Sanes JR 2000 Imaging neuronal subsets in transgenic mice expressing multiple spectral variants of GFP. Neuron 28 41-51. (doi:10.1016/S08966273(00)00084-2)

Guthoff RF, Zhivov A \& Stachs O 2009 In vivo confocal microscopy, an inner vision of the cornea - a major review. Clinical \& Experimental Ophthalmology 37 100-117. (doi:10.1111/j.14429071.2009.02016.x)

Islam MS 2013 Animal models of diabetic neuropathy: progress since 1960s. Journal of Diabetes Research 2013149452 (doi:10.1155/2013/149452)

Published by Bioscientifica Ltc. http://joe.endocrinology-journals.org

DOI: $10.1530 / \mathrm{JOE}-16-0284$
() 2016 Society for Endocrinology Printed in Great Britain 
Ivanusic JJ, Wood RJ \& Brock JA 2013 Sensory and sympathetic innervation of the mouse and guinea pig corneal epithelium. Journal of Comparative Neurology 521 877-893. (doi:10.1002/ cne.23207)

Lauria G, Hsieh ST, Johansson O, Kennedy WR, Leger JM, Mellgren SI, Nolano M, Merkies IS, Polydefkis M, Smith AG, et al. 2010 European Federation of Neurological Societies/Peripheral Nerve Society Guideline on the use of skin biopsy in the diagnosis of small fiber neuropathy. Report of a joint task force of the European Federation of Neurological Societies and the Peripheral Nerve Society. European Journal of Neurology 17 903-912, e944-e909. (doi:10.1111/j.14681331.2010.03023.x

Leppin K, Behrendt AK, Reichard M, Stachs O, Guthoff RF, Baltrusch S, Eule JC \& Vollmar B 2014 Diabetes mellitus leads to accumulation of dendritic cells and nerve fiber damage of the subbasal nerve plexus in the cornea. Investigative Ophthalmology \& Visual Science 55 3603-3615. (doi:10.1167/iovs.14-14307)

Malik RA 2014 Which test for diagnosing early human diabetic neuropathy? Diabetes 63 2206-2208. (doi:10.2337/db14-0492)

Meijering E, Jacob M, Sarria JC, Steiner P, Hirling H \& Unser M 2004 Design and validation of a tool for neurite tracing and analysis in fluorescence microscopy images. Cytometry A 58 167-176. (doi:10.1002/cyto.a.20022)

Muller LJ, Pels L \& Vrensen GF 1996 Ultrastructural organization of human corneal nerves. Investigative Ophthalmology \& Visual Science $\mathbf{3 7}$ 476-488.

Namavari A, Chaudhary S, Sarkar J, Yco L, Patel K, Han KY, Yue BY, Chang JH \& Jain S 2011 In vivo serial imaging of regenerating corneal nerves after surgical transection in transgenic thy1-YFP mice. Investigative Ophthalmology \& Visual Science 52 8025-8032. (doi:10.1167/iovs.11-8332)

Porrero C, Rubio-Garrido P, Avendano C \& Clasca F 2010 Mapping of fluorescent protein-expressing neurons and axon pathways in adult and developing Thy1-eYFP-H transgenic mice. Brain Research $\mathbf{1 3 4 5}$ 59-72. (doi:10.1016/j.brainres.2010.05.061)

Pritchard N, Dehghani C, Edwards K, Burgin E, Cheang N, Kim H, Mikhaiel M, Stanton G, Russell AW, Malik RA, et al. 2015 Utility of assessing nerve morphology in central cornea versus whorl area for diagnosing diabetic peripheral neuropathy. Cornea 34 756-761. (doi:10.1097/ICO.0000000000000447)

Quattrini C, Tavakoli M, Jeziorska M, Kallinikos P, Tesfaye S, Finnigan J, Marshall A, Boulton AJ, Efron N \& Malik RA 2007 Surrogate markers of small fiber damage in human diabetic neuropathy. Diabetes $\mathbf{5 6}$ 2148-2154. (doi:10.2337/db07-0285)

Reichard M, Hovakimyan M, Wree A, Meyer-Lindenberg A, Nolte I, Junghans C, Guthoff R \& Stachs O 2010 Comparative in vivo confocal microscopical study of the cornea anatomy of different laboratory animals. Current Eye Research 35 1072-1080. (doi:10.3109/ 02713683.2010.513796)

Reichard M, Hovakimyan M, Guthoff RF \& Stachs O 2014 In vivo visualisation of murine corneal nerve fibre regeneration in response to ciliary neurotrophic factor. Experimental Eye Research 120 20-27. (doi:10.1016/j.exer.2013.12.015)

Reichard M, Weiss H, Poletti E, Ruggeri A, Guthoff RF, Stachs O \& Baltrusch S 2016 Age-related changes in murine corneal nerves. Current Eye Research 41 1021-1028. (doi:10.3109/02713683.2015.1088952)

Rosenberg ME, Tervo TM, Immonen IJ, Muller LJ, Gronhagen-Riska C \& Vesaluoma MH 2000 Corneal structure and sensitivity in type 1 diabetes mellitus. Investigative Ophthalmology \& Visual Science 41 2915-2921.

Tavakoli M, Quattrini C, Abbott C, Kallinikos P, Marshall A, Finnigan J, Morgan P, Efron N, Boulton AJ \& Malik RA 2010 Corneal confocal microscopy: a novel noninvasive test to diagnose and stratify the severity of human diabetic neuropathy. Diabetes Care 33 1792-1797. (doi:10.2337/dc10-0253)

Tavakoli M, Mitu-Pretorian M, Petropoulos IN, Fadavi H, Asghar O, Alam U, Ponirakis G, Jeziorska M, Marshall A, Efron N, et al. 2013 Corneal confocal microscopy detects early nerve regeneration in diabetic neuropathy after simultaneous pancreas and kidney transplantation. Diabetes 62 254-260. (doi:10.2337/db12-0574)

Winter K, Scheibe P, Kohler B, Allgeier S, Guthoff RF \& Stachs O 2016 Local variability of parameters for characterization of the corneal subbasal nerve plexus. Current Eye Research 41 186-198. (doi:10.3109/ 02713683.2015.1010686)

Yorek MS, Obrosov A, Shevalye H, Lupachyk S, Harper MM, Kardon RH \& Yorek MA 2014 Effect of glycemic control on corneal nerves and peripheral neuropathy in streptozotocin-induced diabetic C57Bl/6J mice. Journal of the Peripheral Nervous System 19 205-217. (doi:10.1111/jns.12086)

Yorek MS, Obrosov A, Shevalye H, Holmes A, Harper MM, Kardon RH \& Yorek MA 2015 Effect of diet-induced obesity or type 1 or type 2 diabetes on corneal nerves and peripheral neuropathy in C57Bl/6J mice. Journal of the Peripheral Nervous System 20 24-31. (doi:10.1111/ jns.12111)

Yu CQ \& Rosenblatt MI 2007 Transgenic corneal neurofluorescence in mice: a new model for in vivo investigation of nerve structure and regeneration. Investigative Ophthalmology \& Visual Science 48 1535-1542. (doi:10.1167/iovs.06-1192)

Ziegler D, Papanas N, Zhivov A, Allgeier S, Winter K, Ziegler I, Bruggemann J, Strom A, Peschel S, Kohler B, et al. 2014 Early detection of nerve fiber loss by corneal confocal microscopy and skin biopsy in recently diagnosed type 2 diabetes. Diabetes 63 2454-2463. (doi:10.2337/db13-1819)

Received in final form 26 August 2016

Accepted 6 September 2016

Accepted Preprint published online 6 September 2016
๑) 2016 Society for Endocrinology Printed in Great Britain
Published by Bioscientifica Ltd. 